12.03

\title{
Экситоны в квантовых ямах на основе ZnO
}

\author{
(C) М.Н. Батаев ${ }^{1}$, Н.Г. Философров ${ }^{1}$, А.Ю. Серов ${ }^{1}$, В.Ф. Агекян ${ }^{1}$, \\ C. Mohrain ${ }^{2}$, В.П. Кочерешко ${ }^{1,3, \uparrow}$ \\ ${ }^{1}$ Санкт-Петербургский государственный университет, \\ Санкт Петербург, Россия \\ ${ }^{2}$ Centre de Recherche sur l'Hetero-Epitaxie et ses Applications-CNRS, Rue Bernard Gregory, \\ Valbonne, France \\ ${ }^{3}$ Физико-технический институт им. А.Ф. Иофффе РАН, \\ Санкт Петербург, Россия \\ ฯ E-mail: Vladimir.Kochereshko@mail.ioffe.ru
}

(Поступила в Редакцию 22 мая 2018 г.)

Детально исследованы спектры отражения и фотолюминесценции структур $\mathrm{ZnO} / \mathrm{Zn}_{0.78} \mathrm{Mg}_{0.22} \mathrm{O}$ с квантовыми ямами $\mathrm{ZnO}$ и толстыми слоями $\mathrm{ZnO}$ и $\mathrm{Zn}_{0.78} \mathrm{Mg}_{0.22} \mathrm{O}$ в зависимости от температуры, интенсивности и длины волны возбуждения. Идентифицированы все наблюдаемые в спектрах линии, установлено, что встроенное электрическое поле не оказывает такого сильного влияния на спектр, как это предполагалось. Повидимому, встроенное поле эффективно экранируется носителями, перешедшими в зоны с уровней доноров и акцепторов. Проведена оценка параметров, определяющих свойства экситона в оксиде цинка.

Работа частично поддержана грантом СПбГУ № 11.37.210.2016.

DOI: 10.21883/FTT.2018.12.47356.143

\section{1. Введение}

Оксид цинка является весьма перспективным полупроводником для различных применений. Его свойства сходны со свойствами нитрида галлия, который уже стал незаменимым материалом в оптоэлектронике. При этом технология изготовления структур на основе $\mathrm{ZnO}$ проще и качество этих структур выше по сравнению с $\mathrm{GaN}$. В последние годы $\mathrm{ZnO}$ активно исследуется в связи с перспективой придания ему новых магнитных свойств.

Как полупроводник $\mathrm{ZnO}$ известен давно [1], наиболее полный обзор свойств $\mathrm{ZnO}$ содержится в диссертации [2]. Однако, в литературе наблюдается сильное разночтение не только в отношении параметров этого материала, но и в отношении идентификации многочисленных линий, наблюдающихся в его спектре излучения, даже в относительно недавних статьях мы находим противоречивые данные.

Причины такого разночтения связаны с тем, что в $\mathrm{ZnO}$, как правило, содержится много примесей, вдобавок к этому в гетероструктурах на основе оксида цинка возможно присутствие встроенного пьезоэлектрического поля, вызванного механическими напряжениями вследствие рассогласования параметров решетки материалов на интерфейсах.

В отличие от классических полупроводниковых структур экситонные линии в спектрах излучения $\mathrm{ZnO}$ слабы по сравнению с линиями примесного происхождения. Вследствие этого интерпретация структуры спектров фотолюминесценции, отражения и пропускания $\mathrm{ZnO}$ вызывает определенные затруднения.

В данной работе мы провели комплексное исследование оптических свойств структуры с квантовыми ямами на основе $\mathrm{ZnO}$ с целью надежно идентифицировать экситонные линии и, по возможности, определить параметры экситонов и встроенного электрического поля.

\section{2. Эксперимент}

Исследованные образцы были выращены методом молекулярно-лучевой эпитаксии на подложках из сапфира. Толщина буферного слоя $\mathrm{ZnO}$, сформированного непосредственно на $c$-плоскости сапфира, составляет $2 \mu \mathrm{m}$. Затем были выращены барьерный слой $\mathrm{Zn}_{0.78} \mathrm{Mg}_{0.22} \mathrm{O}$ толщиной $200 \mathrm{~nm}$, две квантовые ямы $\mathrm{ZnO}$ толщиной $2.7 \mathrm{~nm}$ и $7.1 \mathrm{~nm}$, разделенные барьером $\mathrm{Zn}_{0.78} \mathrm{Mg}_{0.22} \mathrm{O}$ толщиной $200 \mathrm{~nm}$, и покрывающий слой $\mathrm{Zn}_{0.78} \mathrm{Mg}_{0.22} \mathrm{O}$ толщиной $200 \mathrm{~nm}$. Исследовались спектры отражения и фотолюминесценции этой структуры при различных энергиях кванта возбуждающего света, плотностях мощности возбуждения и температурах.

На рис. 1 представлен спектр отражения исследуемой структуры (кривая $a$ ). Контур отражения (1) с энергией $3.65 \mathrm{eV}$, близкой к ширине запрещенной зоны $\mathrm{Zn}_{0.78} \mathrm{Mg}_{0.22} \mathrm{O}$, связан с экситонным резонансом в $\mathrm{Zn}_{0.78} \mathrm{Mg}_{0.22} \mathrm{O}$. Обращает на себя внимание то, что этот контур „перевернут“, то есть с увеличением энергии коэффициент отражения сначала уменьшается, достигает минимума, а затем растет, достигая максимума. Такая форма контура экситонного отражения не является типичной для объемных полупроводников.

Причина „вращения“ контура экситонного отражения подробно обсуждается в монографии [3], она связана с существованием достаточно толстого безэкситонного „мертвого“ слоя в приповерхностной области объемных кристаллов. Переворот контура отражения часто 


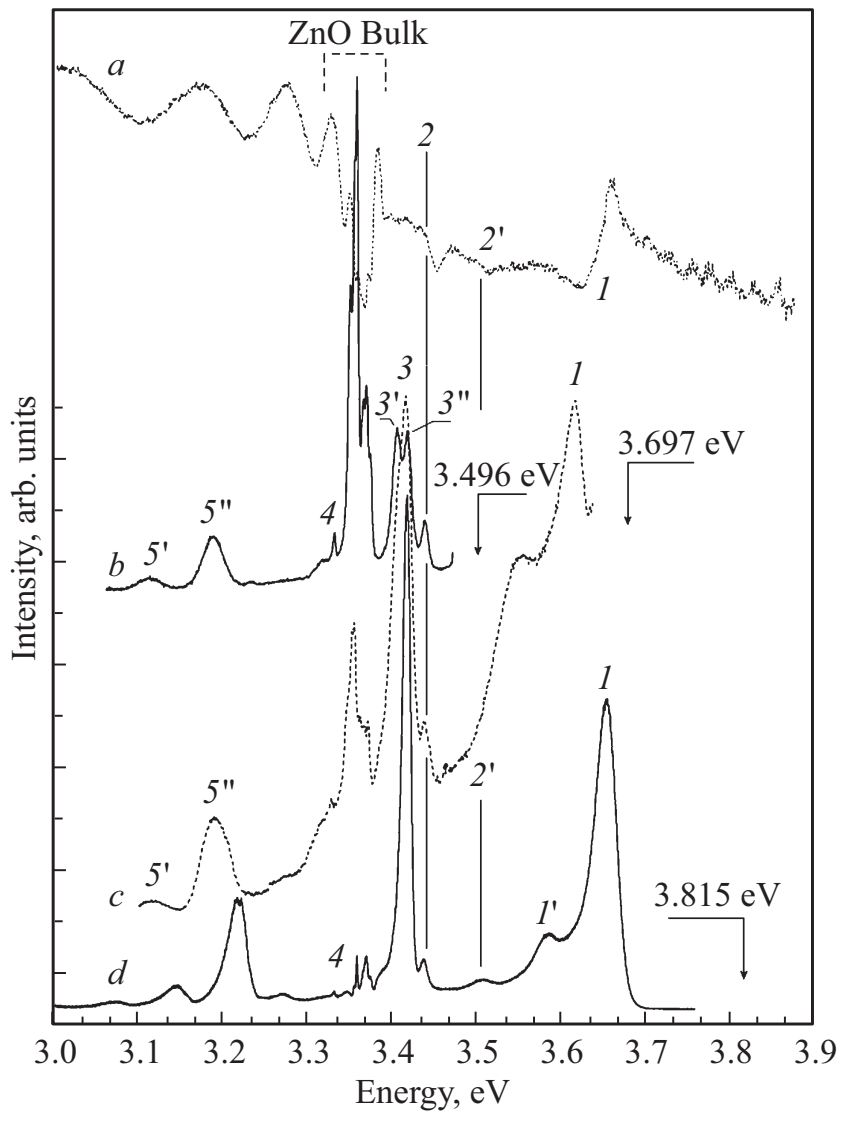

Рис. 1. Оптические спектры структуры $\mathrm{ZnO} / \mathrm{Zn}_{0.78} \mathrm{Mg}_{0.22} \mathrm{O}$ с двумя квантовыми ямами $\mathrm{ZnO}$ шириной 2.7 и $7.1 \mathrm{~nm}$. $a$ - спектр отражения при нормальном падении света; $b-$ спектр фотолюминесценции при возбуждении с энергией $3.503 \mathrm{eV} ; c-$ спектр фотолюминесценции при возбуждении с энергией $3.679 \mathrm{eV} ; d-$ спектр фотолюминесценции при возбуждении с энергией $3.815 \mathrm{eV}$. Линия (1) соответствует экситону в барьерных слоях $\mathrm{Zn}_{0.78} \mathrm{Mg}_{0.22} \mathrm{O}$; линии (2) и (3) соответствуют экситону и триону в узкой квантовой яме; область обозначенная (Bulk $\mathrm{ZnO}$ ) соответствует экситонам в буферном слое $\mathrm{ZnO}$; линия (4) соответствует экситону в широкой квантовой яме; линии (5) соответствуют донорно акцепторным парам в слое $\mathrm{ZnO}$.

наблюдается и в структурах с квантовыми ямами, он вызван интерференцией света в безэкситонном барьерном слое [4], который является аналогом „мертвого“ слоя в объемных полупроводниках. Пользуясь результатами работы [4], можно легко оценить толщину безэкситонного слоя. Для контура отражения, необходимо, чтобы набег фазы при прохождении светом безэкситонного слоя составлял, это условие соответствует толщине слоя порядка $32 \mathrm{~nm}$ при величине фонового показателя преломления [5].

Подобный переворот контура экситонного отражения наблюдался и в структурах на основе $\mathrm{GaN}$ [6], где образование мертвого слоя связано с экранированием в приповерхностной области встроенного пьезоэлектрического поля, возникшего вследствие рассогласования постоянных решетки материалов подложки и различных слоев гетероструктуры. Вдали от поверхности это поле экранируется свободными носителями и напряженность его невелика (см. рис. 4). Вблизи поверхности наличие электрического поля приводит к ионизации экситона и сдвигу его резонансной энергии. Такая ситуация возможна и в нашем случае, поскольку структура соединений $\mathrm{Zn}_{0.78} \mathrm{Mg}_{0.22} \mathrm{O}$ и $\mathrm{GaN}$ одинакова и их диэлектрические параметры близки.

В работе [6] для нитрида галлия было установлено, что величина ионизирующего экситон электрического поля составляет порядка $1.46 \cdot \cdot^{5} \mathrm{~V} / \mathrm{cm}$, а толщина безэкситонного слоя равна $45 \mathrm{~nm}$ при концентрации свободных носителей $2.2 \cdot 10^{17} \mathrm{~cm}^{-3}$ (см. также [3]). Концентрация свободных электронов определяется из условия, что электростатическое поле экранируется на длине безэкситонного слоя. Напряженность поля, ионизирующего экситон, можно оценить по аналогии с атомом водорода. Для атома водорода эта величина составляет $F_{1 H}=2.57 \cdot 10^{9} \mathrm{~B} / \mathrm{cm}$, тогда для экситона $F_{1}\left(\mu / m_{0}\right)^{2} \varepsilon_{\infty}^{-2} \varepsilon_{0} F_{1 H} \approx 2.4 \cdot 10^{5} \mathrm{~V} / \mathrm{cm}$ (здесь $\mu$ приведенная масса экситона, $\mu=0.22 m_{0}$, где $m_{0}-$ масса электрона, $\varepsilon_{0}$ и $\varepsilon_{\infty}-$ статическая и высокочастотная диэлектрические проницаемости). В работе [6] для нитрида галлия использовались значения $\varepsilon_{0}=8.9$ и $\varepsilon_{\infty}=5.35$.

Поскольку в $\mathrm{ZnO}$ радиус экситона составляет лишь около $20 \mathrm{~nm}$, при рассмотрении экранирования кулоновского поля в экситоне следует использовать высокочастотное значение диэлектрической постоянной $\varepsilon_{\infty}=6.7$, а для экранирования статического поля низкочастотное значение $\varepsilon_{0}=8$. Используя результаты работы [6] и учитывая различие параметров $\mathrm{ZnO}$ и $\mathrm{GaN}$, получаем, что длина экранирования приповерхностного поля в $\mathrm{ZnO}$ составляет $33 \mathrm{~nm}$ при концентрации электронов $3 \cdot 10^{17} \mathrm{~cm}^{-3}$. Эта величина совпадает с дебаевской длиной экранирования при той же концентрации электронов и температуре $2 \mathrm{~K}$.

Следует заметить, что полученное значение концентрации электронов является оценочным, поскольку мы предполагаем, что приповерхностное поле полностью ионизует экситон. Это условие не является обязательным, даже в заметно меньших полях экситонное затухание оказывается достаточно большим, чтобы можно было говорить о „мертвом“ слое. Кроме того, мы считали, что носители полностью экранируют приповерхностное поле, что также не обязательно - небольшое поле может сохраниться в объемной области структуры.

Для описания формы контура отражения экситона в слоях $\mathrm{Zn}_{0.78} \mathrm{Mg}_{0.22} \mathrm{O}$ и определения экситонных параметров можно воспользоваться общепринятым выражением для зависимости диэлектрической проницаемости от частоты света $\omega$ вблизи экситонного резонанса

$$
\varepsilon(\omega)=\varepsilon_{b}+\frac{\varepsilon_{b} \omega_{L T}}{\omega_{T}-\omega-i \Gamma} .
$$

Здесь: $\omega_{L T}-$ величина продольно-поперечного расщепления, $\varepsilon_{b} \approx 8-$ фоновая диэлектрическая проницаемость, $\omega_{T}$ резонансная частота экситона, $\Gamma-$ экситонное затухание. Так как $\omega_{L T} / \Gamma \ll 1$, пространственной дисперсией можно пренебречь. 
Зная амплитуду и ширину контура экситонного отражения, можно определить величину продольно поперечного расщепления и диссипативное затухание экситона [7]. Ширина контура отражения в нашем случае составляет $36 \mathrm{meV}$, она равна удвоенной величине диссипативного затухания $\Gamma$, амплитуда контура составляет $0.2 R_{0}$ (20\% от усредненного значения коэффициента отражения от поверхности $\left.\mathrm{Zn}_{0.78} \mathrm{Mg}_{0.22} \mathrm{O}\right)$. Здесь $R_{0}$ - коэффициент отражения света от поверхности, $R_{0}=\left(\frac{n-1}{n+1}\right)^{2}, n-$ показатель преломления света. Отсюда получаем, что величина продольно поперечного расщепления $\omega_{L T}$ объемного экситона в $\mathrm{Zn}_{0.78} \mathrm{Mg}_{0.22} \mathrm{O}$ составляет около $2 \mathrm{meV}$. Эта простая оценка хорошо согласуется с данными для экситона серии А в ZnO [1].

Ниже по энергии от линии отражения экситона в барьерном слое наблюдается линия отражения (2) (рис. 1, кривая $a$ ). Мы относим эту линию, имеющую энергию $3.444 \mathrm{eV}$ и ширину около $10 \mathrm{meV}$, к основному состоянию экситона в квантовой яме шириной $2.7 \mathrm{~nm}$. Линию отражения $\left(2^{\prime}\right)$ с энергией $3.51 \mathrm{eV}$ можно отнести к возбужденному состоянию этого экситона. Ширина этой линии равна $20 \mathrm{meV}$, что заметно превышает ширину линии основного состояния экситона. По амплитуде и ширине линии (2) можно оценить величину радиационного затухания экситона в квантовой яме [7]. В случае узкой квантовой ямы радиационная ширина линии $\Gamma_{0}$ связана с продольно-поперечным расщеплением соотношением

$$
\Gamma_{0}=k \omega_{L T}^{\mathrm{eff}} a .
$$

Здесь $\Gamma_{0}$ - радиационное затухание экситона в квантовой яме, $a$ - ширина квантовой ямы, $k=\frac{\omega}{c} n-$ волновой вектор света, $\omega_{L T}^{\mathrm{eff}}-$ эффективная величина продольно поперечного расщепления, для узкой квантовой ямы $k a \ll 1$.

Мы получаем, что радиационное затухание экситона в квантовой яме $\Gamma_{0} \approx 0.6 \mathrm{meV}$, что соответствует величине эффективного продольно-поперечного расщепления $\omega_{L T}$ eff $\approx 4 \mathrm{meV}$. Эта величина представляется разумной для экситона в квантовой яме, так как с уменьшением ширины ямы радиационная ширина экситонной линии возрастает. Так, например, в узких квантовых ямах GaAs радиационная ширина линии $\Gamma_{0}$ может увеличиться в 3 раза по сравнению с ее значением в объемным кристалле [8].

Из спектрального положения второго резонанса в спектре отражения можно оценить энергию связи экситона как $90 \mathrm{meV}$. Это примерно в 1.5 раза больше энергии связи для объемного экситона в $\mathrm{ZnO}$ [9].

В длинноволновой области спектра отражения (она обозначена на рис. 1 как $\mathrm{ZnO}$ Bulk) наблюдаются интерференционные особенности, сбегающиеся к экситонному резонансу в буферном слое ZnO. Такая картина типична для отражения света от тонких слоев полупроводника вблизи экситонных резонансов [10]. Наличие интерференционной картины позволяет воссоздать вид дисперсионных кривых поляритонов в слое $\mathrm{ZnO}$, определить энергию экситонного резонанса, а также величины затухания и продольно-поперечного расщепления.

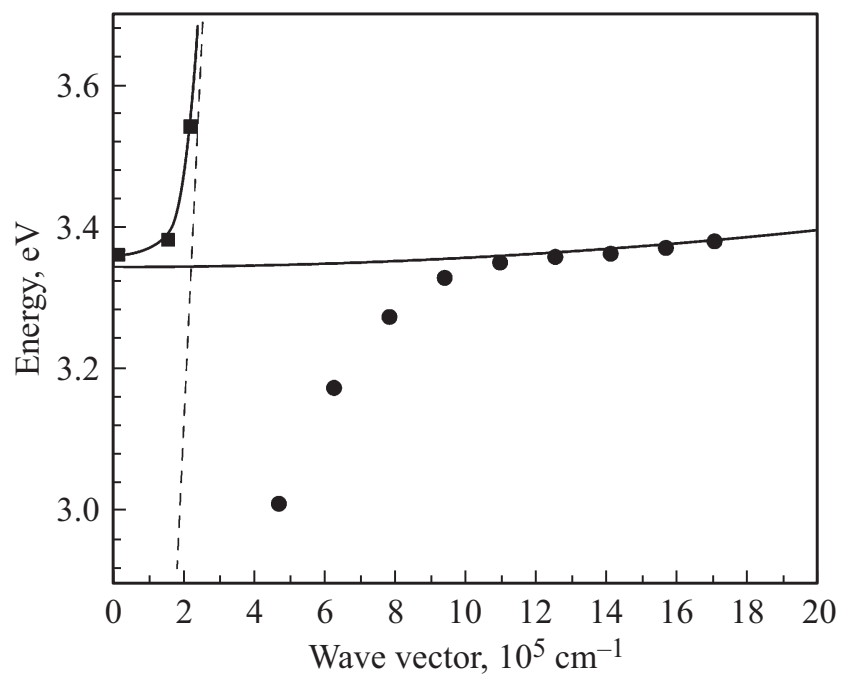

Рис. 2. Дисперсионные кривые поляритонов, построенные на основе интерференционных особенностей спектра отражения аналогично работе [10]. Использованы следующие параметры: трансляционная масса экситона $0.9 m_{0}$, фоновая диэлектрическая проницаемость $\varepsilon=8$.

По известной толщине слоя $\mathrm{ZnO} d=2 \mu \mathrm{m}$ мы можем определить номера интерференционных особенностей $N$ используя условие квантования $k_{N} d=\pi N$, где $k_{N}-$ волновой вектор экситона. Далее, сопоставляя каждой особенности ее энергию $E_{N}$ и волновой вектор $k_{N}$, можно построить дисперсионную кривую поляритона (рис. 2). При этом построении мы использовали величину эффективной массы экситона равную $0.9 m_{0}$ и фоновую диэлектрическую проницаемость $\varepsilon=8$.

Максимум коэффициента отражения в спектре структуры с энергией $3.382 \mathrm{meV}$ приблизительно соответствует частоте продольного экситона $\omega_{L}$ в ZnO. Действительно, как видно из формулы (1), вещественная часть диэлектрической проницаемости обращается в нуль на продольной частоте: $\varepsilon\left(\omega_{L}\right)$. Следовательно, коэффициент отражения на границе слоев и $\mathrm{Zn}_{0.78} \mathrm{Mg}_{0.22} \mathrm{O}$ на этой частоте достигает максимума. Поперечная частота экситона в данном спектре, вообще говоря, не выделяется, однако, построив дисперсионные кривые, можно аппроксимировать их к частоте экситонного резонанса. Из аппроксимации, приведенной на рис. 2, следует, что энергия экситонного резонанса в слоях составляет $E_{\mathrm{ZnO}}=3.375 \mathrm{eV}$, что совпадает с табличным значением [9] для $\mathrm{ZnO}$.

Линия отражения от квантовой ямы с шириной $7.1 \mathrm{~nm}$ налагается на интерференционную картину от слоев $\mathrm{ZnO}$, и ее трудно идентифицировать по спектрам отражения. Она наблюдается лишь в виде небольшого „сбоя“ интерференционной картины на энергии $3.331 \mathrm{eV}$. Следует иметь в виду, что линия фотолюминесценции поляритонов обычно расположена ниже по энергии относительно резонансной частоты экситона и проявляется в области максимального экситон фотонного смешивания. 
Для прояснения картины мы зарегистрировали спектры фотолюминесценции в зависимости от длины волны и плотности оптического возбуждения (рис. 1, спектры $b, c, d)$. Спектр фотолюминесценции при надбарьерном возбуждении с энергией кванта $3.815 \mathrm{eV}$ показан на рис. 1 , спектр $d$.

Область спектра от $3.55 \mathrm{eV}$ до $3.70 \mathrm{eV}$, лежащая выше резонансных энергий экситонов в узкой квантовой яме (2), относится к барьерным слоям $\mathrm{Zn}_{0.78} \mathrm{Mg}_{0.22} \mathrm{O}$. В этой области наблюдается интенсивный пик фотолюминесценции, энергия которого $3.65 \mathrm{eV}$ (линия (1) на спектре $d$ рис. 1) соответствует линии отражения экситона (спектр $a$ на рис. 1).

На расстоянии $63 \mathrm{meV}$ от линии (1) находится линия $\left(1^{\prime}\right)$, которая соответствует фононному повторению линии (1). В спектре отражения эта линия отсутствует, что естественно для фононных повторений.

При фотовозбуждении в область коротковолнового края полосы (1) с энергией кванта $3.679 \mathrm{eV}$ линии (1) и $\left(1^{\prime}\right)$ (спектр $c$, рис. 1) сдвигаются в сторону меньших энергий на величину около $30 \mathrm{meV}$ относительно их положения в спектре $d$ ). При этом другие линии, соответствующие излучению квантовой ямы $2.7 \mathrm{~nm}$ и объемного экситона $\mathrm{ZnO}$, не сдвигаются.

Столь сильный сдвиг трудно объяснить проявлением эффекта Штарка на свободном экситоне в толстых слоях $\mathrm{Zn}_{0.78} \mathrm{Mg}_{0.22} \mathrm{O}$. Как показывают исследования атомов в электрическом поле, длинноволновый штарковский сдвиг для атома водорода не может превышать 10\% от его энергии связи [11], более того, в сильных электрических полях длинноволновый сдвиг сменяется коротковолновым. Следовательно, для свободного экситона в барьерных слоях сдвиг, связанный с электрическим полем не должен превышать $6 \mathrm{meV}$.

В наших спектрах длинноволновый сдвиг линии (1) наблюдается только при изменении энергии кванта возбуждающего света, и отсутствует при увеличении интенсивности возбуждения. Следовательно, этот сдвиг не является ни штарковским сдвигом, ни сдвигом, связанным с энергетической релаксацией по непрерывному хвосту локализованных экситонных состояний $\mathrm{Zn}_{0.78} \mathrm{Mg}_{0.22} \mathrm{O}$ [12]. Мы считаем, что этот сдвиг связан с локализацией экситона на кластерах $\mathrm{ZnO}$ в твердом растворе $\mathrm{Zn}_{0.78} \mathrm{Mg}_{0.22} \mathrm{O}$. Действительно, постоянные решетки $\mathrm{ZnO}$ и $\mathrm{MgO}$ сильно различаются и, следовательно, в твердом растворе может быть энергетически выгодным образование кластеров (исследованию структуры твердого раствора $\mathrm{Zn}_{0.78} \mathrm{Mg}_{0.22} \mathrm{O}$ будет посвящена отдельная статья).

Ниже экситонного резонанса (2) в узкой квантовой яме наблюдается интенсивная линия (3) с энергией $3.416 \mathrm{eV}$. Мы полагаем, что эта линия связана с проявлением трионов и/или связанных экситонов. Энергию связи триона в квантовой яме можно оценить в $20-25 \%$ от энергии связи экситона [13]. Полученная выше концентрация электронов $3 \cdot 10^{17} \mathrm{~cm}^{-3}$ соответствует тем концентрациям, при которых наблюдается яркая люминесценция трионов в структурах с несколько меньшей энергией связи экситонов и трионов [14]. Следует заметить, что трион сложно отличить от экситона, связанного на нейтральном доноре. Единственное отличие состоит в том, что энергия связи экситона на доноре несколько больше, чем энергия связи триона. В работе [15] было показано, что в квантовых ямах на основе CdTe энергия связи экситона на нейтральном доноре в 1.5 раза больше энергии связи свободного триона.

При возбуждении с энергией кванта $3.678 \mathrm{eV}$ в область барьерного экситона отношение интенсивностей линий (2) и (3) меняется в пользу линии (2), при этом у линии (3) появляется длинноволновое крыло $\left(3^{\prime}\right)$.

При подбарьерном возбуждении с энергией $3.502 \mathrm{eV}$ соотношение интенсивностей линия (2) и (3) еще сильнее изменяется в пользу линии (2), а линия (3) превращается в дублет (3) и $\left(3^{\prime}\right)$. Мы считаем, что коротковолновая компонента этого дублета связана с рекомбинацией триона, а длинноволновая компонента с рекомбинацией экситона на нейтральном доноре.

Такое перераспределение интенсивностей линий люминесценции можно объяснить следующим образом. При надбарьерном возбуждении рождается много электронов и дырок, которые разделяются встроенным полем. Часть этих электронов и дырок собираются в квантовых ямах и связывается в экситоны. Благодаря большей подвижности электроны попадают квантовые ямы легче, чем дырки. В результате этого в квантовых ямах присутствуют экситоны и свободные электроны, что и приводит к образованию трионов. Вероятность связывания экситона и электрона очень велика даже при относительно небольшой концентрации электронов, кроме того, образование трионов энергетически выгодно. Благодаря этому линии люминесценции трионов обычно значительно интенсивнее линий экситонов [16].

Вследствие однородного фонового легирования структуры в ее квантовых ямах могут присутствовать как нейтральные доноры и акцепторы, так и свободные электроны. При подбарьерном возбуждении электроннодырочные пары рождаются прямо в квантовой яме, и концентрация добавочных электронов в ней невелика. Поэтому при образовании носителей непосредственно в квантовой яме интенсивности линий свободных экситонов и экситонов, связанных на нейтральных донорах, увеличиваются по сравнению с интенсивностью трионных линий.

Ниже резонансной энергии объемного экситона в $\mathrm{ZnO}$ наблюдаются две линии фотолюминесценции с энергиями $3.368 \mathrm{eV}$ и $3.358 \mathrm{eV}$, интенсивность которых модулируется короткопериодной интерференцией. Период этой интерференции совпадает с периодом интерференции в спектре отражения в том же спектральном диапазоне. Естественно связать эти две линии с рекомбинацией экситонов в толстом слое $\mathrm{ZnO}$. Действительно, интерференционная картина в оптических спектрах кристаллов всегда связана пропусканием света на границе кристалла, в нашем случае это граница между $\mathrm{Zn}_{0.78} \mathrm{Mg}_{0.22} \mathrm{O}$. 
Таким образом, интерференционная картина должна наблюдаться как в спектрах отражения и пропускания, так и в спектрах люминесценции [17]. Следовательно, источником излучения в этой области спектра является слой $\mathrm{ZnO}$.

Мы связываем коротковолновую линию $3.368 \mathrm{eV}$ с рекомбинацией экситона в слое $\mathrm{ZnO}$, а линию $3.358 \mathrm{eV}-$ с рекомбинацией триона. Соотношение интенсивностей этих линий изменяется в пользу трионов при переходе от подбарьерного возбуждения к надбарьерному. При увеличении интенсивности возбуждения происходит аналогичное перераспределение их интенсивностей - интенсивность длинноволновой линии растет значительно быстрее интенсивности коротковолновой, что совпадает с поведением линий, относящихся к излучению из узкой квантовой ямы. Это также подтверждает интерпретацию этих линий как аннигиляцию экситона и триона.

В спектре люминесценции присутствует еще одна слабая линия (4) с энергией $3.331 \mathrm{eV}$, которую мы относим к рекомбинации экситона в широкой квантовой яме $\mathrm{ZnO}$.

Длинноволновые линии в спектре фотолюминесценции ниже $3.3 \mathrm{eV}$ вероятнее всего связаны с туннельной рекомбинацией донорно-акцепторных пар в толстом слое $\mathrm{ZnO}$. С увеличением интенсивности возбуждения эти линии сдвигаются в длинноволновую сторону, при переходе от надбарьерного возбуждения к подбарьерному их положение изменяется на $30 \mathrm{meV}$.

Энергии линий люминесценции, соответствующих рекомбинации донорно-акцепторных пар, определяются формулой

$$
E(r)=E_{g}-\left(E_{A}+e_{D}\right)+\frac{e^{2}}{\varepsilon r} .
$$

Здесь: $E_{A}-$ энергия дырки на акцепторе, $E_{D}-$ энергия электрона на доноре, $E_{g}$ - ширина запрещенной зоны, $r$ - расстояние между донором и акцептором. При увеличении интенсивности оптического возбуждения все больше доноров и акцепторов оказываются заселенными, и среднее расстояние между заселенными центрами уменьшается. В результате, как следует из формулы (3), энергия оптического перехода увеличивается, что и наблюдается в наших спектрах. Очевидно, что расстояние $r$ между донором и акцептором не может быть меньше боровского радиуса, минимальное расстояние между донором и акцептором может составлять около двух боровских радиусов. В таком случае энергия взаимодействия донора и акцептора составляет примерно $30 \mathrm{meV}$, именно такой сдвиг и наблюдается в нашем эксперименте.

Мы провели также исследование температурной зависимости спектров фотолюминесценции в условиях надбарьерного и подбарьерного возбуждения. С увеличением температуры весь спектр сдвигается в длинноволновую сторону в соответствии с изменением ширины запрещенной зоны, при этом происходит значительное перераспределение интенсивности между различными

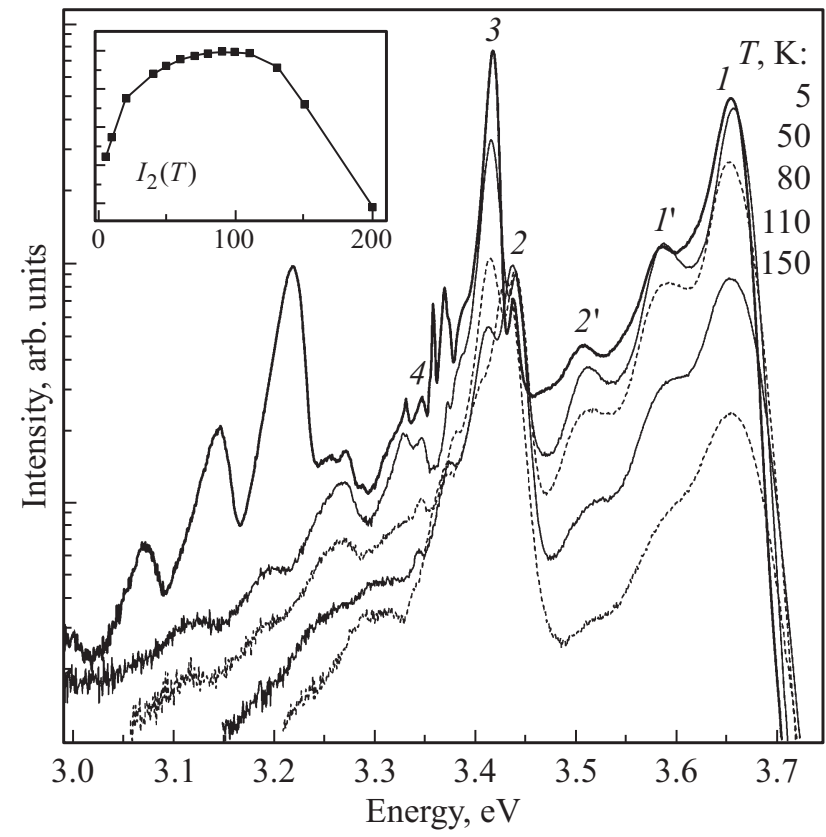

Рис. 3. Температурные зависимости спектров фотолюминесценции структуры $\mathrm{ZnO} / \mathrm{Zn}_{0.78} \mathrm{Mg}_{0.22} \mathrm{O}$ при возбуждении с энергией $3.815 \mathrm{eV}$, построенные в полулогарифмическом масштабе. Номера линий соответствуют их обозначениям на рис. 1. На вставке приведена температурная зависимость интенсивности линии (2) экситона в узкой квантовой яме. Видно, что интенсивность этой линии растет вплоть до $100 \mathrm{~K}$.

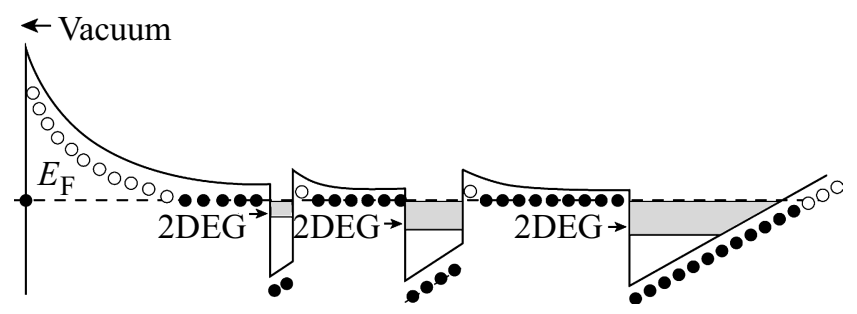

Рис. 4. Зонная диаграмма исследованной структуры $\mathrm{ZnO} / \mathrm{Zn}_{0.78} \mathrm{Mg}_{0.22} \mathrm{O}$. Пунктир - уровень Ферми; пустые кружки - ионизированные доноры; заполненные кружки - заряженные доноры.

компонентами спектра (рис. 3). Полосы, связанные с донорно-акцепторной рекомбинацией, исчезают уже при температуре около $20 \mathrm{~K}$, линии связанных экситонов и трионов наблюдаются вплоть до $50-80 \mathrm{~K}$.

Линии излучения (1), (2) и (4) наблюдаются даже при температурах выше $150 \mathrm{~K}$. Более того, линия (2) усиливается с ростом температуры по мере того, как интенсивности линий связанных экситонов и трионов падают. Это подтверждает экситонную природу линий (1), (2) и (4).

В наших образцах мы не обнаружили заметного проявления встроенного электрического поля. Отсутствие сильного электрического поля можно объяснить следующим образом (рис. 4). В структуре присутствуют 
как доноры, так и акцепторы, избыточная концентрация доноров $N_{D}-N_{A}=3 \cdot 10^{17} \mathrm{~cm}^{-3}$. На поверхности структуры уровень Ферми пинингуется поверхностными состояниями, в объеме уровень Ферми совпадает с уровнем доноров. В результате вблизи поверхности возникает обедненный слой положительно заряженных донорных центров. Электроны с этих центров переходят в квантовые ямы, и в зону проводимости $\mathrm{ZnO}$. Они частично экранируют встроенное поле и обеспечивают образование трионов в квантовых ямах и буферном слое.

Таким образом, у поверхности имеется избыточный положительный заряд, а в квантовых ямах и на границе $\mathrm{Zn}_{0.78} \mathrm{Mg}_{0.22} \mathrm{O}$ и буферного слоя $\mathrm{ZnO}$ возникает отрицательно заряженные слои электронов, пришедших с доноров. Зная концентрацию доноров, можно грубо оценить поле в таком „конденсаторе“ как $t \cdot 10^{5} \mathrm{~V} / \mathrm{cm}$, что близко к величинам, приведенным в статье [5] и других работах. Это поле компенсирует пьезоэлектрическое поле в барьерных слоях. В результате суммарное поле в структуре уменьшается. При этом поле в самих ямах не только не компенсируется, но складывается с пъезоэлектрическим полем. Это приводит с тому, что линия экситона в широкой яме (4) оказывается сдвинутой в длинноволновую сторону относительно линии объемного экситона в $\mathrm{ZnO}$ на $\sim 40 \mathrm{meV}$. Оценка штарковского сдвига [7] в квантовой яме шириной $7.1 \mathrm{~nm}$ в поле дает величину $60 \mathrm{meV}$. В узкой квантовой яме $2.7 \mathrm{~nm}$, энергия размерного кантования носителей больше энергии электрического поля и штарковский сдвиг мал. Таким образом, имеем хорошее согласование всех величин, учитывая, что оценка поля как $7 \cdot 10^{5} \mathrm{~V} / \mathrm{cm}$ является несколько завышенной, так как модель „конденсатора“ не вполне подходит к нашей ситуации.

\section{3. Заключение}

В работе детально исследованы спектры отражения и фотолюминесценции структуры, содержащей толстые слои $\mathrm{ZnO}$ и $\mathrm{Zn}_{0.78} \mathrm{Mg}_{0.22} \mathrm{O}$ и квантовые ямы $\mathrm{ZnO}$, в зависимости от температуры, интенсивности возбуждения и длины волны возбуждения. В спектрах удалось идентифицировать все наблюдаемые линии, установлено, что встроенное электрическое поле не оказывает сильного влияния на энергии оптических переходов, за исключением энергии экситона в широкой квантовой яме $7.1 \mathrm{~nm}$. Показано, что встроенное пьезоэлектрическое поле экранируется носителями, которые переходят в зоны с доноров и акцепторов. Проведена оценка параметров, описывающих экситон.

\section{Список литературы}

[1] D.G. Thomas. J. Phys. Chem. Solids 15, 86 (1960); J.J. Hopfield. J. Phys. Chem. Solids 15, 97 (1960).

[2] M.R. Wagner Fundamental properties of excitons and phonons in ZnO: A spectroscopic study of the dynamics, polarity, and effects of external fields. Doctor der Naturwissenschaft, Berlin (2010).
[3] В.А. Киселев, Б.В. Новиков, А.Е. Чередниченко. Экситонная спектроскопия приповерхностной области полупроводников. Изд-во СПбГУ (2003).

[4] Е.Л. Ивченко, П.С. Копьев, В.П. Кочерешко, И.Н. Уральцев, Д.Р. Яковлев, С.В. Иванов. ФТП 22, 5, 784 (1988).

[5] C. Mohrain, T. Bretagnon, P. Lefebre, X. Tang, P. Valvin, T. Guillet, D. Gil, T. Taliercio, M. Teisseire-Doninelli, B. Vinter, C. Deparis. Phys. Rev. B 71, 241305(R) (2005).

[6] Д.А. Андроников. Спектры отражсения эпитаксиальных слоев GaN. Бакалаврская работа. СПГУ (2002).

[7] E.L. Ivchenko. Optical Spectroscopy of Semiconductor Nanostructures. Alpha Science Int., Harrow, UK (2005).

[8] E.L. Ivchenko, V.P. Kochereshko, P.S. Kopev, V.A. Kosobukin, I.N. Uraltsev, D.R. Yakovlev. Solid State Commun. 70, 5, 529 (1989).

[9] Landolt Börnstein database. Springer (2000).

[10] В.А. Киселев, Б.С. Разбирин, И.Н. Уральцевю. Письма в ЖЭТФ 18, 8, 504 (1973).

[11] V.V. Kolosov. J. Phys. B. 20, 2359 (1987).

[12] C.F. Klingshirn. Semiconductor Optics. Springer (1997).

[13] G.V. Astakhov, D.R. Yakovlev, V.P. Kochereshko, W. Ossau, W. Faschinger, J. Puls, F. Henneberger, S.A. Crooker, Q. McCulloch, D. Wolverson, N.A. Gippius, A. Waag. Phys. Rev. B 65, 16335 (2002).

[14] G.V. Astakhov, D.R. Yakovlev, V.P. Kochereshko, W. Ossau, J. Nürnberger, W. Faschinger, G. Landwehr. Phys. Rev. B 60, R8485 (1999).

[15] F. Bassani, S. Tatarenko, K. Saminadayar, N. Magnea, R.T. Cox, A. Tardot, C. Grattepain. J. Appl. Phys. 72, 2927 (1992).

[16] V.P. Kochereshko, G.V. Astakhov, D.R. Yakovlev, W. Ossau, G. Landwehr, T. Wojtowicz, G. Karczewski, J. Kossut. Phys. Status Solidi B 201, 345 (2000).

[17] E. Gross, S. Permogorov, V. Travnikov, A. Selkin. Solid State Commun. 10, 11, 1071 (1972).

Редактор К.В. Емцев 